\title{
Reduced-Rate Retransmissions for Spread-Spectrum Packet Radio Multimedia Networks
}

\author{
Lei Zhou, Student Member, IEEE, Yu-Dong Yao, Senior member, IEEE, \\ Harry Heffes, Fellow, IEEE, and Ruifeng Zhang, Member, IEEE
}

\begin{abstract}
A reduced-rate retransmission (RRR) scheme is proposed for improving the throughput performance of spreadspectrum packet radio networks. The scheme takes advantages of the available multi-rate scalable source coding techniques. It assumes that several versions of a data packet with different sizes (number of information bits) are available. The transmission of a packet starts from its full-size version. If the full-size version is not correctly received, its half-size version is used in the retransmission. If further retransmissions are needed, the quarter-size version and so on are used. The shrunk packets are transmitted either in a minislot if the processing gain is kept the same, or occupying a slot duration by increasing the processing gain proportionally. In both cases, the effective signal to interference ratio for a packet is increased. As a result, the system throughput is improved. Theoretical and numerical results are provided in this paper which illustrate the throughput improvement. Another advantage of the proposed RRR scheme is that the packet-size reduction provides finer granules for link adaptation. Therefore, it is especially suitable for multimedia applications for which codes of variable rate for the source data are available and which can tolerate gracefully degraded quality of service. The performance of the proposed scheme in fading channels is also addressed.
\end{abstract}

Index Terms - Spread spectrum, packet radio, link adaptation.

\section{INTRODUCTION}

$\mathbf{S}^{\mathrm{p}}$ PREAD-SPECTRUM PACKET RADIO (SSPR) network is a random access system that uses spread-spectrum signaling. It has attracted intensive interests for decades due to its flexibility in providing multiple access communications amongst users of diverse traffic sources [1]-[6]. Besides its initial applications in military communications, SSPR has also been incorporated in commercial systems, such as the third generation $(3 \mathrm{G})$ cellular system [7].

The SSPR system does not suffer from the classical collision problem like the narrow-band random access system does. It allows correct reception of a packet from contending

Manuscript received April 22, 2003; revised February 25, 2004 and April 4, 2004; accepted March 14, 2005. The associate editor coordinating the review of this paper and approving it for publication was A. Sheikh.

Lei Zhou, formerly of the Department of Electrical and Computer Engineering, Stevens Institute of Technology, is now with Motorola. (e-mail: leizhou@motorola.com).

Yu-dong Yao, and Harry Heffes are with the Department of Electrical and Computer Engineering, Stevens Institute of Technology, Hoboken, NJ 07030 USA (e-mail: yyao@steven.edu; hheffes@stevens.edu).

Ruifeng Zhang is with the Department of Electrical and Computer Engineering, Drexel University, Philadelphia, PA 19104 USA (e-mail: rzhang@ece.drexel.edu). His work was partly supported by ONR (Grant N00014-03-1-0123.

Digital Object Identifier 10.1109/TWC.2006.04012 transmissions due to the unique spreading sequence used by each user. However, excess multi-access interference may cause too many bit errors and impair the system throughput. There have been a good amount of effort to improve the packet reception in interference.

Power capture phenomenon, that is, a strong signal overrides interfering ones and captures the receiver, which arises due to the near-far effect and channel fading, has been explored to improve the throughput performance of random access systems [8]. Unlike for narrow-band Aloha systems that power capture benefits throughput under any traffic conditions, the effect of power capture on SSPR systems is negative in a low traffic region but positive in a high traffic region [9]. An adaptive power control scheme has been proposed in [9] which switches the power control mechanism on or off according to the traffic rate.

Due to the exchangeable roles of power and bandwidth in spread-spectrum systems, power control can also be implemented through adjusting the processing gain [10]-[15]. Usually, fixed chip rate (i.e., radio bandwidth) is assumed. Increasing/decreasing the processing gain means decreasing/increasing the bit rate. The resulting dynamics between the processing gain and the traffic load may lead to instability as indicated in [10] and [11]. A chip-rate adaptation scheme has also been proposed [16]. However, the complexity of the transceiver for variable chip-rate spread-spectrum signaling renders it hardly practical.

SSPR systems also possess the unique delay capture phenomenon, which is due to the correlation property of the pseudo-noise spreading sequence [17]. An optimal arrival time distribution to take advantage of the delay capture effect has been investigated [18] for given channel conditions and the number of contending users. However, control of arrival time is not always an easy task for packet networks given the asynchronous nature of packet transmissions and random variations of propagation delay.

Little attention has been paid to the problem of retransmitting the corrupted packets, which is an important component of random access systems. In most studies, simple random backoff procedures are implemented and the corrupted packets are repeated until they go through or are discarded after several repeats. The problem with the backoff strategy is that it incurs long delay and cannot sustain stability. From an information-theoretic viewpoint, a packet failure is due to excess multi-access interference and the user should reduce its information rate to maintain the reliability of transmissions. In 
other words, rate reduction is needed when there are too many users accessing the channel. The fixed-chip-rate processing gain adaptation schemes in [10], [12]-[15] provides certain rate control mechanisms. However, they are only suitable for session-level adaptations. They require traffic load parameters (packet arrival rate or the number of contending users) in determining the transmission parameters (processing gain and power), which are usually difficult to measure and feedback by either individual users or the central base station on a packetby-packet basis or even every few packets. The power [9] and arrival time [18] adaptation schemes are also more or less at the session-level because traffic parameters are needed there.

The processing gain adaptation scheme proposed in [11] is a packet (medium access control) level adaptation scheme. It bases the adjustment of processing gain for each packet on the number of retransmissions of the previous packet. Compared to session-level adaptation, packet-level adaptation is able to respond to traffic and channel variations more quickly. However, the scheme in [11] does not provide rate adaptation mechanisms for retransmissions of corrupted packets. It is expected that rate adaptation in retransmissions will offer more flexibility.

In this paper, we propose and analyze a rate adaptation scheme for retransmissions of corrupted packets. Specifically, we assume that a data packet comes in several versions of different sizes. The transmission of it starts from its full-size version. If the full-size version is not correctly received, a version of smaller size is used in the retransmission. If the smaller version fails again, an even smaller one is retransmitted. This procedure continues until the packet goes through successfully or the version with the minimum size is used. The rational for such a reduced-rate retransmission (RRR) scheme lies in that the reduction in packet size reduces traffic load offered to the channel when the packet traffic becomes high. Another explanation may be that reducing the packet size reduces effective multi-access interference when there are too many colliding packets so that the success probability of the packet reception and the system throughput can be increased.

The proposed RRR scheme takes advantage of the widely available multi-rate source coding techniques, e.g., [19], [20], which provide scalable resolutions of the information sources in response to different link quality. These techniques are very suitable for multimedia services for which gracefully degraded quality of service is tolerable.

In the rest of the paper, we first give the system model in Section II. Then in Section III we present the proposed RRR scheme. In Section IV we analyze the throughput and delay performance of the proposed schemes. Numerical results of theoretical analyses and simulations are given in Section V. Conclusions are drawn in Section VI.

\section{SYSTEM MODEL}

\section{A. Source Encoding and Scaling}

We consider the communications among a group of multimedia users. We assume that the users use certain scalable multi-rate coding scheme to encode their sources such that the code can be scaled down to represent the source with different resolutions (Fig. 1). As a result, a data packet comes

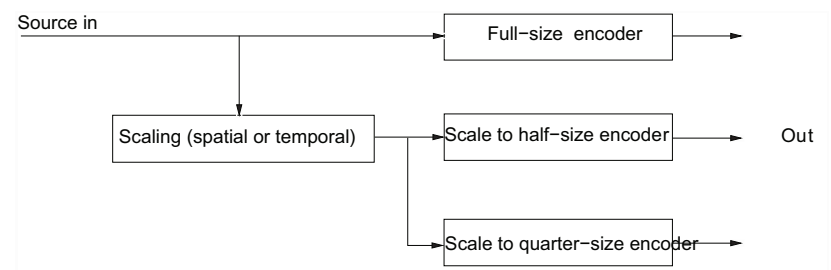

Fig. 1. Source encoder model.

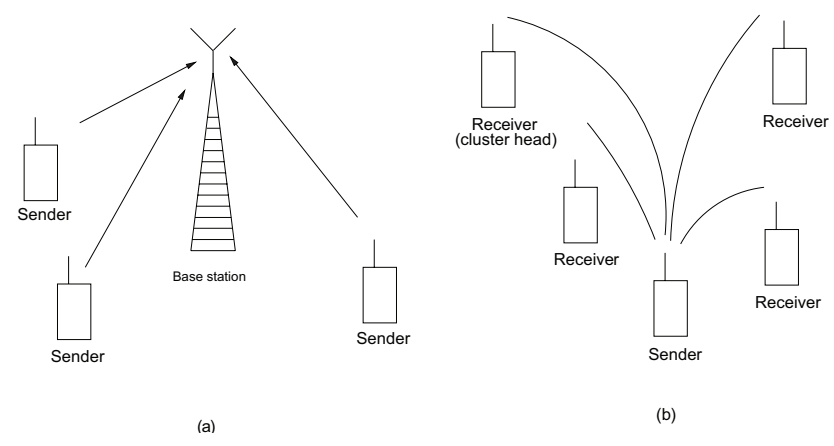

Fig. 2. SSPR system model: (a) mobile to base station, (b) mobile to mobile.

in several versions of different sizes: full-size, half-size and so on. A simple coding scheme can be down-sampling the analog source or using coarser quantization. It can also be implemented by discarding less significant discrete cosine transform (DCT) or wavelet transform (WT) coefficients. This procedure can be also be optimized according to the ratedistortion function [21]. However, the specific encoding and decoding algorithms are not within the scope of this paper.

\section{B. Spread-Spectrum Packet Radio}

The considered communication system is a one-hop SSPR system, in which packets are transmitted from one user to another via a central station. By central station, we mean either the base station in a cellular network (Fig. 2(a)) or a cluster head in an ad-hoc network (Fig. 2(b)). Only the uplink communications (from users to the central station) is studied. The uplink channel is a spreaded slotted-Aloha channel. The length of the channel slot is equal to that of a full-size packet (with highest encoding rate) and slot synchronization among users is assumed. We further assume that at the end of each slot the central station is able to feedback whether or not a packet transmitted in the past slot is correctly received, i.e., positive acknowledgement (ACK) or negative acknowledgement (NACK), and that the ACK/NACK is received instantly and with no errors. The NACKed packets are subject to retransmissions.

\section{The REDUCED-RATE RETRAnsmission SCHEME}

The basic protocol of the RRR scheme is as following. A user packet is firstly transmitted using its full-size version. If it is NACKed, its half-size version is used for retransmission in the succeeding slot. Similarly, if the half-size version is NACKed again, the quarter-size version will be used for the 


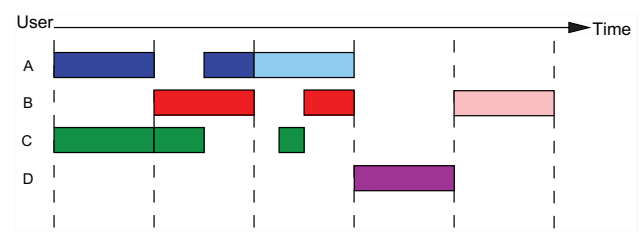

(a)

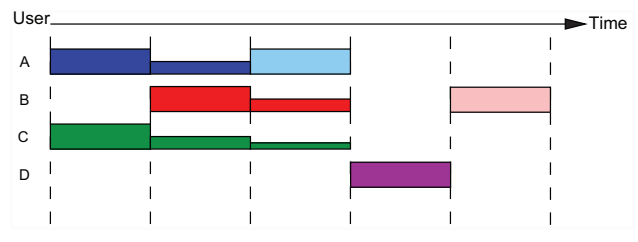

(b)

Fig. 3. Packet transmission snapshots: (a) Approach 1, (b) Approach 2; blocks of the same shade represents the same packet (re)transmitted using its different-sized versions and the height of the blocks means different processing gains.

second retransmission. The procedure may continue until the smallest version of the packet is used or the maximum allowed number of retransmissions is reached.

In order to accommodate the small-size packets into the channel slot structure, the SSPR system allows two approaches:

Approach 1: The spreading factor for the half-size version is the same as the full-size so that the transmitted packet is only of half the length of the channel slot. It is designed that the transmission of the half-size packet starts randomly from either the beginning or the middle of the channel slot. Similarly, the starting point for transmission of a quarter-size packet is either the beginning or one of the quarter points of the slot. The similar rule applies to all other smaller-size packets.

Approach 2: The spreading factor increases proportionally as the packet size reduces in successive retransmissions. The half-size version is spreaded two times more than the full-size, and the quarter-size is spreaded four times more, and so on. Therefore, the transmitted packet is always of the same length as the channel slot.

The two reduced-rate retransmission approaches are different only in the signaling format: Approach 1 keeps the bit duration fixed while Approach 2 keeps the packet duration fixed. In Approach 1, synchronization of packet (re)transmissions at the middle and quarter points of the channel slots is required. This is usually achieved using the minislot technique. In Approach 2 the chip rate is fixed. Doubling the spreading factor is through doubling the bit duration. Snapshots of the reduced-rate retransmission procedure is shown in Fig. 3.

Notice that the proposed retransmission schemes do not implement the random backoff mechanism though they are able to. The essence of the proposed schemes is to reduce the multi-access interference through reducing the information rate of users so as to increase the channel throughput, instead of trying to avoid collisions. This idea is especially meaningful for spreaded random access channels in which packets are correctly received from interference that are well controlled. It will be shown by simulations that RRR method outperforms the conventional backoff method without rate reduction in terms of channel throughput. Another advantage of the instance retransmission in RRR is that it incurs a relatively shorter delay, a key requirement for delay-sensitive applications. Nevertheless, it is easy to incorporate the random backoff mechanisms into the RRR schemes. The study of such a combination however, is not pursued in this paper.

As a final remark, we want to indicate that the $50 \%$ reduction of the packet size in each retransmission is not a necessity but just a working example of the RRR method. Actually, other step size in the reduction can also be used. A small step size in packet size reduction may avoid under-utilizing the channel capacity while a large step size may respond to the channel changes faster. Implementation-wise, the minislot length or the spreading factor multiples need to be changed to address the multiple packet size considerations. However, these variations will not be explored in this paper and they can be easily accommodated in the framework developed in this paper with minor extensions.

\section{PERFormance AnAlysis}

In this section, we analytically evaluate the performance of the proposed RRR protocol. For the sake of conceptual clarity and mathematical tractability, we study the case that only one retransmission is allowed for a packet, i.e., a packet is discarded if its half-size version fails in the transmission. The one-retransmission scheme is also a sensible choice for delaysensitive applications like voice and video in which any excess retransmission delay is intolerable. In addition, as it will be shown in later sections, one retransmission is practically able to approach the bit-rate capacity of the channel and, therefore, more retransmissions are not always necessary.

\section{A. Packet Throughput and Bit Throughput}

We first study the throughput performance of the reducedrate retransmission scheme. There are two types of throughput that are of interest: packet throughput and bit throughput. The former is defined as the average number of packets successfully transmitted in one packet duration (channel slot) and the latter the average number of bits going through in one bit duration. Denote with $W(m, n)$ the probability that there are $m$ full-size and $n$ half-size packets being transmitted in a time slot. Denote also with $S(k, l \mid m, n)$ the probability that $k$ out of $m$ full-size packets and $l$ out of $n$ half-size packets are successfully received in a time slot. Then the two types of throughput can be expressed as

Packet throughput:

$$
R_{P}=\sum_{m=0}^{\infty} \sum_{n=0}^{\infty} \sum_{k=0}^{m} \sum_{l=0}^{n}(k+l) S(k, l \mid m, n) W(m, n),
$$

Bit throughput:

$$
R_{B}=\frac{1}{L} \sum_{m=0}^{\infty} \sum_{n=0}^{\infty} \sum_{k=0}^{m} \sum_{l=0}^{n}\left(k+\frac{l}{2}\right) S(k, l \mid m, n) W(m, n),(2)
$$

respectively, where $L$ is the full packet size (also the slot length) measured in number of bits. We see that the packet throughput is always higher than the bit throughput, $R_{P} \geq$ $R_{B}$. 
The two types of throughput are both important measures of quality of service (QoS) for multimedia applications. For transmission of packetized voice, video, picture, etc., the user can usually tolerate certain quality degradation caused by coarse coding rather than silence due to packet loss or delay. In this case, packet throughput is a good indicator of the system performance. While, the bit throughput represents the utmost information rate provided by the system and, therefore, is a more thorough characterization of QoS.

To compute $R_{P}$ and $R_{B}$, we need $S(k, l \mid m, n)$ and $W(m, n)$, which are to be derived in the next two sections.

\section{B. Packet Success Probability}

The value of $S(k, l \mid m, n)$ is determined by the packet error rate (PER), which, in turn, depends on specific modulation and coding schemes of the packets and channel fading conditions. As an initial step, we consider a non-fading (or perfectly power-controlled) channel in this section and the fading cases will be considered in section IV-E. We follow the Gaussian approximation approach [22], [23] to study the bit error rate (BER) of the spread-spectrum system. However, more precise BER models for spread-spectrum systems, e.g., [24], are equally applicable to our analyses in the sequel. They are not pursued here for the sake of succinctness in describing the essential idea. Moreover, the basic behavior the BER characteristics as against to the signal-to-interference ratio (SIR) obtained using different models are roughly the same. Therefore, more complicated models will not have different impacts on the throughput.

For Approach 1, referring to Fig. 3(a), we note that a fullsize packet interferes with all other full-size packets and all, but not necessarily simultaneously, half-size packets; and a half-size packet interferes with all full-size packets and other half-size packets in the same half-slot. If the half-size packets are uniformly distributed in the two half-slots, which is true when the half-size packets starts from the beginning or the middle of the slot with equal probability, then on average half of the half-size packets interfere with the full-size packets and with each other at a time. As a result, any packet, full or halfsize, experiences interference from other $m+n / 2-1$ packets. An example of the interference between the full-size and halfsize packets is the second slot from the right in Fig. 3(a), where one $(m=1)$ full-size packet (red) experiences interference from one of the two $(n=2)$ half-size packets at two subslots. The SIR for the full-size and half-size packets are the same,

$$
r_{f}(m, n)=r_{h}(m, n)=\frac{G}{m+n / 2-1}, \quad \text { for Approach } 1
$$

where $G$ is the processing gain. In (3), the effect of noise has been omitted in light of the interference-limit nature of multi-access systems.

For Approach 2, referring to Fig. 3(b), all packets are interfering to each other, i.e., any packet experiences interference from other $m+n-1$ packets; but full-size and Half-size packets have different SIR,

$$
\begin{aligned}
r_{f}(m, n) & =\frac{G}{m+n-1}, \\
r_{h}(m, n) & =\frac{2 G}{m+n-1}, \quad \text { for Approach 2 }
\end{aligned}
$$

since they have different processing gains.

The following analyses are common to both Approach 1 and 2. Using Gaussian approximation, the bit error rate (BER) can be expressed as a function of the SIR

$$
P_{e, f / h}(m, n)=Q\left(\sqrt{r_{f / h}(m, n)}\right)
$$

for the full-size and half-size packets, respectively, where $Q(x)=\int_{x}^{\infty} 1 / \sqrt{2 \pi} \exp \left(-t^{2} / 2\right) d t$. The PER depends on the BER and the available error-correcting code. If protection of up to $d$ bit-errors is provided, the PER can be expressed as

$$
\begin{array}{r}
P_{E, f}(m, n)=1-\sum_{j=0}^{d}\left(\begin{array}{c}
L \\
j
\end{array}\right) P_{e, f}^{j}(m, n) \\
{\left[1-P_{e, f}(m, n)\right]^{L-j},} \\
P_{E, h}(m, n)=1-\sum_{j=0}^{d}\left(\begin{array}{c}
L / 2 \\
j
\end{array}\right) P_{e, h}^{j}(m, n) \\
{\left[1-P_{e, h}(m, n)\right]^{L / 2-j}}
\end{array}
$$

for the full-size packets and half-size packets, respectively. We have assumed the same amount of bit-error protection for both full-size and half-size packets. This may results in more overhead to the half-size packets.

With the PER, the probability that $k$ out of $m$ full-size packets and $l$ out of $n$ half-size packets are successfully received in a slot is written as

$$
\begin{aligned}
S(k, l \mid m, n)= & \left(\begin{array}{c}
m \\
k
\end{array}\right)\left(\begin{array}{c}
n \\
l
\end{array}\right)\left[1-P_{E, f}(m, n)\right]^{k} P_{E, f}^{m-k}(m, n) \\
& {\left[1-P_{E, h}(m, n)\right]^{l} P_{E, h}^{n-l}(m, n) . }
\end{aligned}
$$

In later analyses, we will also need the marginal distribution of the number of successful full-size packet, which can be obtained as

$$
S(k \mid m, n)=\left(\begin{array}{c}
m \\
k
\end{array}\right)\left[1-P_{E, f}(m, n)\right]^{k} P_{E, f}^{m-k}(n),
$$

representing the probability that $k$ full-size packets are successfully received, irrespective of the number of successful half-size packets, given $m$ full-size and $n$ half-size packets being transmitted.

\section{Packet Traffic Analysis}

The distribution of the number of full-size and half size packets in a slot, $W(m, n)$, is a steady-state expression. Suppose that there are $M_{t}$ full-length packets and $N_{t}$ halfsize packets in the $t$-th slot. The joint mass function of $M_{t}$ and $N_{t}$ is denoted with $p_{M_{t} N_{t}}(m, n)$. Then, $W(m, n)$ is the steady-state of $p_{M_{t} N_{t}}(m, n)$,

$$
W(m, n)=\lim _{t \rightarrow \infty} p_{M_{t} N_{t}}(m, n) .
$$

The full-size packets in a slot are those generated by the users during the previous slot. We assume that the generation of packets by all users can be modeled as a Poisson process of rate $\lambda$ packets per slot time. Therefore, the marginal mass function of the number of full-size packets in the $t$-th slot, $M_{t}$, is

$$
p_{M_{t}}(m)=\frac{\lambda^{m}}{m !} e^{-\lambda} .
$$




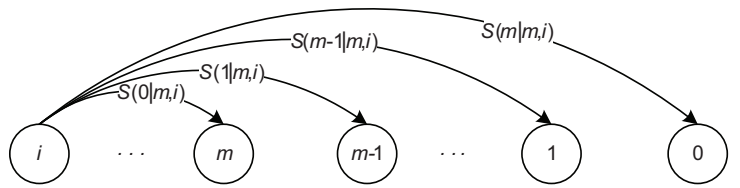

Fig. 4. Transition diagram of $N_{t-1}=i$ to $N_{t}=j$ conditioned on $M_{t-1}=$ $m$.

Note that the expression is independent of $t$, so is the steadystate expression.

The half-size packets in a slot are part of the full-size packets in the previous slot that have failed in transmission. It is easy to infer that the number of half-size packets in the $t$-th slot, $N_{t}$, solely depends on the number of full-size and half-size packets in the $(t-1)$-st slot, $M_{t-1}$ and $N_{t-1}$, and is independent of the number of full-size packets in the $t$-th slot, $M_{t}$, due to the memoryless property of Poisson processes. Therefore, the joint mass function $p_{M_{t} N_{t}}(m, n)$ can be broken down as the product of the two marginal mass functions,

$$
p_{M_{t} N_{t}}(m, n)=p_{M_{t}}(m) p_{N_{t}}(n),
$$

and, in the steady state,

$$
W(m, n)=p_{M_{t}}(m) \lim _{t \rightarrow \infty} p_{N_{t}}(n) .
$$

To obtain the steady-state mass function $\lim _{t \rightarrow \infty} p_{N_{t}}(n)$, we need to analyze the process $N_{t}$. $N_{t}$ is a Markov chain and its state transition probability can be written as

$$
P\left[N_{t}=j \mid N_{t-1}=i\right]=\sum_{m=0}^{\infty} S(m-j \mid m, i) p_{M_{t-1}}(m),
$$

where $p_{M_{t-1}}(m)$ is the mass function of the number of fullsize packets, $M_{t-1}$, in the $(t-1)$-st slot and is given in (10), and $S(m-j \mid m, i)$ is the probability that $m-j$ out of $m$ full-size packets are successfully transmitted given that there are $m$ full-size and $i$ half-size packets, defined in (8). (13) is based on the fact that there exist $j$ half-size packets in the $t$-th slot if $m-j$ out of $m$ full-size packets in the $(t-1)$ st slot have been successfully received. The statistical averaging over $M_{t-1}$ is to get an average transition probability for $N_{t}$. The state transition of $N_{t-1}=i$ to $N_{t}=j$ conditioned on $M_{t-1}=m$ is shown in Fig. 4 .

With the state transition probability in (13) for $N_{t}$, obtaining the steady-state mass function of $N_{t}$ is straightforward. Constructing a state transition matrix $\boldsymbol{T}$ with its $(i, j)$-th entry equal to $P\left[N_{t}=j \mid N_{t-1}=i\right]$, and defining $\pi_{n}=$ $\lim _{t \rightarrow \infty} p_{N_{t}}(n)$ and $\boldsymbol{\pi}=\left[\pi_{0}, \pi_{1}, \ldots\right]$, the steady-state mass function of $N_{t}$ can be solved from the linear equations

$$
\pi T=\pi
$$

together with the normalization condition $\sum_{n=0}^{\infty} \pi_{n}=1$.

As a summary, we give the procedure to compute the packet and bit throughput using the derivations in the above. With a given packet length $L$, processing gain $G$ and the errorcorrection capability $d$, one can compute $S(k, l \mid m, n)$ and $S(k \mid m, n)$ for any $m, n, k$ and $l$, according to (7) and (8). With a given packet arrival rate $\lambda$, one can compute $p_{M_{t}}(m)$ according to (10). Then using $S(k \mid m, n)$ and $p_{M_{t}}(m)$, one can compute (13) and (14) to get $\lim _{t \rightarrow \infty} p_{N_{t}}(n)$. With $p_{M_{t}}(m)$ and $\lim _{t \rightarrow \infty} p_{N_{t}}(n)$, one can compute $W(m, n)$ from (12). Finally, $R_{P}$ and $R_{B}$ in (1) and (2) can be computed using $S(k, l \mid m, n)$ and $W(m, n)$. They are functions of the packet size $L$, the processing gain $G$, the error-correction capability $d$, and the traffic rate $\lambda$. Numerical examples of this computation procedure will be given in the next section.

\section{Delay and Packet Drop Rate}

With the derivations in the above, we can also find the average packet delay and packet drop rate. Suppose that a test packet is first transmitted in a slot, say the $t$-th slot, using its full-size version. It will be successfully received with probability $1-P_{E, f}\left(M_{t}, N_{t}\right)$ and fail with probability $P_{E, f}\left(M_{t}, N_{t}\right)$. If it fails, the half-size version is transmitted in the $(t+$ 1)st slot. The second transmission will be successful with probability $1-P_{E, h}\left(M_{t+1}, N_{t+1}\right)$ and fail with probability $P_{E, h}\left(M_{t}, N_{t}\right)$. If we define the packet delay as the number of transmissions needed for a packet to go through successfully, the delay is 1 with probability $1-P_{E, f}\left(M_{t}, N_{t}\right)$ and is 2 with probability $P_{E, f}\left(M_{t}, N_{t}\right)\left[1-P_{E, h}\left(M_{t+1}, N_{t+1}\right)\right]$. Therefore, conditioned on $M_{t}, N_{t}, M_{t+1}$ and $N_{t+1}$, the average delay of a test packet can be written as

$$
\begin{array}{r}
D\left(M_{t}, N_{t}, M_{t+1}, N_{t+1}\right)= \\
\frac{1+P_{E, f}\left(M_{t}, N_{t}\right)-2 P_{E, f}\left(M_{t}, N_{t}\right) P_{E, h}\left(M_{t+1}, N_{t+1}\right)}{1-P_{E, f}\left(M_{t}, N_{t}\right) P_{E, h}\left(M_{t+1}, N_{t+1}\right)}
\end{array}
$$

The presence of the denominator is because only successfully transmitted packets are counted in computing the delay. Averaging (15) over the distribution of $M_{t}, N_{t}, M_{t+1}$ and $N_{t+1}$ and letting $t \rightarrow \infty$ we obtain the average delay as

$$
\begin{array}{r}
D=\lim _{t \rightarrow \infty} \sum_{m 1=1}^{\infty} \sum_{n_{1}=0}^{\infty} \sum_{m_{2}=0}^{\infty} \sum_{n_{2}=1}^{m_{1}} D\left(m_{1}, n_{1}, m_{2}, n_{2}\right) p_{M_{t}}\left(m_{1}\right) \\
p_{N_{t}}\left(n_{1}\right) p_{M_{t+1}}\left(m_{2}\right) p_{M_{t+1} \mid M_{t}, N_{t}}\left(n_{2} \mid m_{1}, n_{1}\right) \text {. (16) }
\end{array}
$$

In (16) we have used the fact that $M_{t}, N_{t}$ and $M_{t+1}$ are independent of each other and that $M_{t+1}$ soly depends on $M_{t}$ and $N_{t}$. The mass functions $p_{M_{t}}\left(m_{1}\right)$ and $p_{m_{t+1}}\left(m_{2}\right)$ are in (10) and the conditional mass function $p_{M_{t+1} \mid M_{t}, N_{t}}\left(n_{2} \mid m_{1}, n_{1}\right)=$ $S\left(m_{1}-n_{2} \mid m_{1}, n_{1}\right)$. They are all independent of $t$, so are steady-state mass functions. The mass function $p_{N_{t}}\left(n_{1}\right)$ in steady-state is derived in (14).

If the half-size version of the test packet fails again, it will be dropped. The probability of dropping will be

$$
\begin{aligned}
P_{\text {drop }}\left(M_{t}, N_{t},\right. & \left.M_{t+1}, N_{t+1}\right)= \\
& P_{E, h}\left(M_{t}, N_{t}\right) P_{E, h}\left(M_{t+1}, N_{t+1}\right),
\end{aligned}
$$

conditioned on $M_{t}, N_{t}, M_{t+1}$ and $N_{t+1}$. The unconditioned packet dropping rate in steady-state is

$$
\begin{aligned}
P_{\text {drop }}= & \lim _{t \rightarrow \infty} \sum_{m_{1}=1}^{\infty} \sum_{n_{1}=0}^{\infty} \sum_{m_{2}=0}^{\infty} \sum_{n_{2}=1}^{m 1} P_{\text {drop }}\left(m_{1}, n_{1}, m_{2}, n_{2}\right) \\
& p_{M_{t}}\left(m_{1}\right) p_{N_{t}}\left(n_{1}\right) p_{M_{t+1}}\left(m_{2}\right) \\
& p_{M_{t+1} \mid M_{t}, N_{t}}\left(n_{2} \mid m_{1}, n_{1}\right) .
\end{aligned}
$$




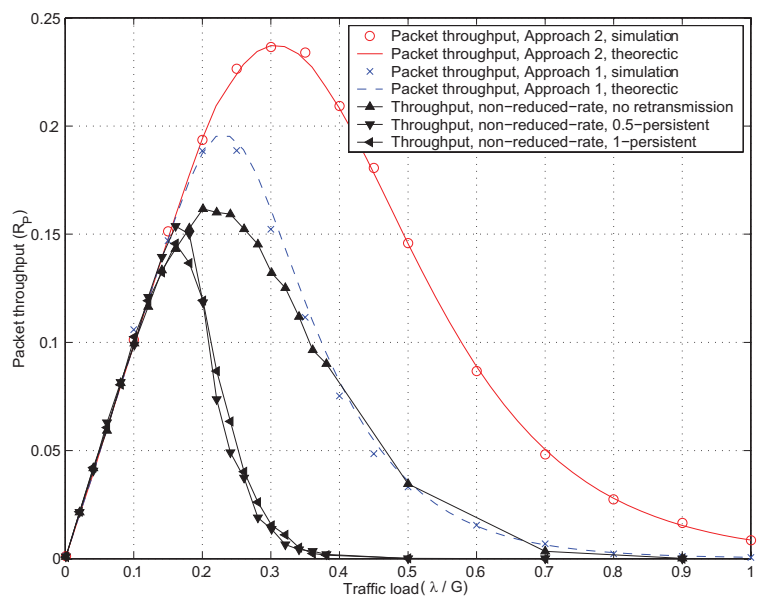

(a)

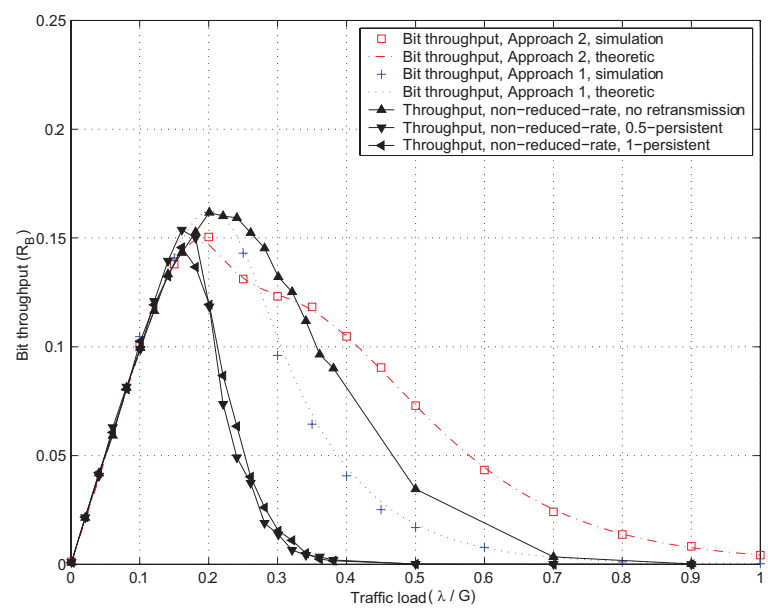

(b)

Fig. 5. Comparison of the reduced-rate retransmission and non-reduced-rate retransmission: (a) packet throughput, (b) bit throughput.

\section{E. Effect of Channel Fading}

In Section IV-B, we have assumed non-fading channels in deriving the PER. However, fading is unavoidable in wireless systems. In this section we extend our analysis to incorporate the effects of channel fading. Generally speaking, channel fading manifests itself in the PER expression, through which it affects the throughput, delay and packet drop rate. Therefore, only the PER analysis in Section IV-B need to be extended.

In order to analyze the channel fading effect, we first need a proper model for the fading. We use the Rayleigh fading model, i.e., the received power $\alpha$ of the faded signal is an exponentially distributed random variable with density function

$$
f(\xi)=\frac{1}{A} e^{-\xi / A},
$$

where $A$ is the average received power. We assume that fading is independent from one user to another and from one packet to another but is static within a slot duration (block fading).

With the presence of the channel fading, the expression of the SIR (for a packet indexed as the $i$-th) becomes

$$
r_{i}=\frac{\alpha_{i} G_{i}}{\sum_{j=1, j \neq i}^{K} \alpha_{j}}
$$

where $G_{i}$ and $\alpha_{i}$ are the processing gain and received power for the $i$-th packet and $K$ is the total number of packets. (20) is a general expression applies to both of the two reduced-rate retransmission approaches and for both full-size and half-size packets. For Approach $1, G_{i}=G$ and $K=m+n / 2$ for all the packets. For Approach 2, $K=m+n$ for all the packets while $G_{i}=G$ for full-size packets and $G_{i}=2 G$ for half-size packets. It may be noted that for Approach 1 $K=m+n / 2$ may not be an integer. However, it turns out that the analytical results in the sequel also applies to non-integer $K$. The requirement that $K$ is an integer is just a working assumption. It can also be explained that certain round up is performed such that an upper bound of $K$ is used.

According to the assumptions made in the previous paragraph, $\alpha_{i}$ 's are independent and identically distributed random variables with exponential density function given in (19). Then, the distribution of the SIR, $r_{i}$, can be derived as following:

$$
\begin{aligned}
& F_{r_{i}}(\xi)=P\left[r_{i} \leq \xi\right]=P\left[\frac{\alpha_{i}}{\sum_{j=1, j \neq i}^{K} \alpha_{j}} \leq \frac{\psi}{G_{i}}\right] \\
= & \int_{0}^{\infty} \cdots \int_{0}^{\infty} \int_{0}^{\frac{\xi_{i}}{G_{i}} \sum_{j=1, j \neq i}^{K} \xi_{j}} f\left(\xi_{i}\right) \\
= & \prod_{0}^{K} \cdots \int_{0=1, j \neq i}^{\infty}\left(1-e^{-\frac{\xi_{i}}{G_{i}}} \frac{\sum_{j=1, j \neq i}^{K} \alpha_{j}}{A}\right) \\
= & 1-\left[\int_{0}^{\infty} e^{-\left(\frac{\xi_{i}}{G_{i}}+1\right) \frac{\xi_{j}}{A}} d \xi_{j}\right]^{K-1} d \xi_{i} d \xi_{1} \cdots d \xi_{K} \\
= & 1-\frac{\prod_{j=1, j \neq i}^{K}}{\left(\xi_{i} / G_{i}+1\right)^{K-1}} \cdot
\end{aligned}
$$

We see that the final expression of (21) allows non-integer $K$.

In the context of the two reduced-rate retransmission schemes, the distribution of the SIR for full-size and half-size packets are

$F_{r_{f}}(\xi)=F_{r_{h}}(\xi)=1-\frac{1}{\left(\frac{\xi_{i}}{G+1}\right)^{m+n / 2-1}}$, for Approach 1

and

$$
\begin{aligned}
& F_{r_{f}}(\xi)=1-\frac{1}{\left(\xi_{i} / G+1\right)^{m+n-1}}, \\
& F_{r_{h}}(\xi)=1-\frac{1}{\left(\xi_{i} / 2 G+1\right)^{m+n-1}}, \text { for Approach 2, }
\end{aligned}
$$

respectively.

The procedure to obtain the BER and PER in the fading environment is largely the same as that in the non-fading case 


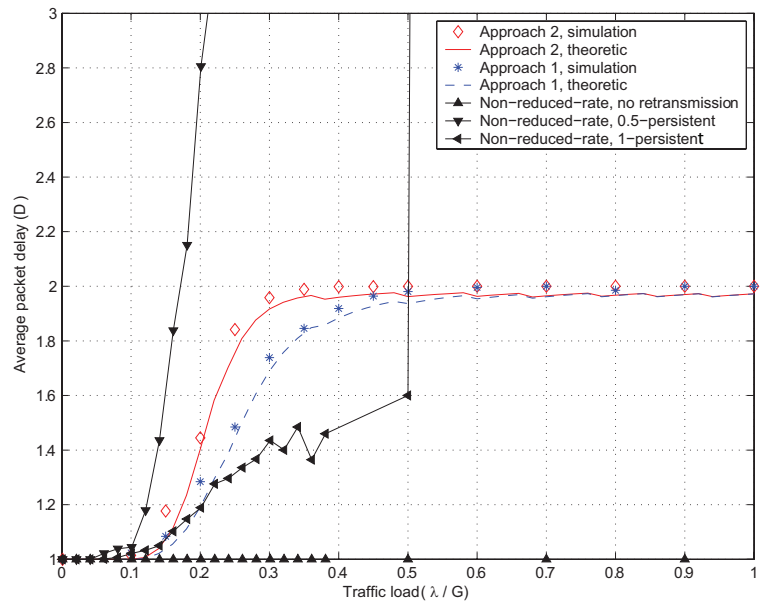

Fig. 6. Comparison of the reduced-rate retransmission and non-reduced-rate retransmission: Average delay.

in Section IV-B, except that averages over the distribution of the SIR is needed. Since block fading has been assumed, all bits in a packet have the same SIR and BER. we can write the BER as

$$
P_{e, f \mid h}\left(r_{f} \mid h\right)=Q\left(\sqrt{r_{f \mid h}}\right) .
$$

Then the PER can be written as

$P_{E, f}=\int_{0}^{\infty}\left\{1-\sum_{j=0}^{d} P_{e, f}^{j}(\xi)\left[1-P_{e, f}(\xi)\right]^{L-j}\right\} d F_{r_{f}}(\xi)$,
$P_{E, h}=\int_{0}^{\infty}\left\{1-\sum_{j=0}^{d} P_{e, h}^{j}(\xi)\left[1-P_{e, h}(\xi)\right]^{L / 2-j}\right\} d F_{r_{h}}(\xi)$.

(25) can be substituted in to (7) and (8) for computing the throughput, delay and packet drop rate.

Finally, we would like to indicate that even though Rayleigh fading model has been used in our analysis, the analytical framework does not exclude other fading models such as Ricean and Nakagami fading.

\section{Analytical and Simulation Results}

In this section, we provide some numerical examples of the analyses developed in the previous sections, as well as simulation results to demonstrate the performance improvement due to the RRR method. A slotted Aloha network is simulated. The packet traffic is generated by potentially infinite number of users according to a Poisson process of rate $\lambda$. We run the simulated network for 10000 channel slots for each given traffic rate. The empirical throughput, delay and packet drop rate are computed based on the statistics of the total number of successful packets the delay of each of them, and the total number of dropped packets during the whole simulation period. The signaling is direct-sequence spreadspectrum. Random sequences are used. The processing gain is $G=31$. Matched filter receivers are used for each transmitted packet. The full-size packet has a length of $L=128$ bits. A virtual error-correction code is assumed which provides a

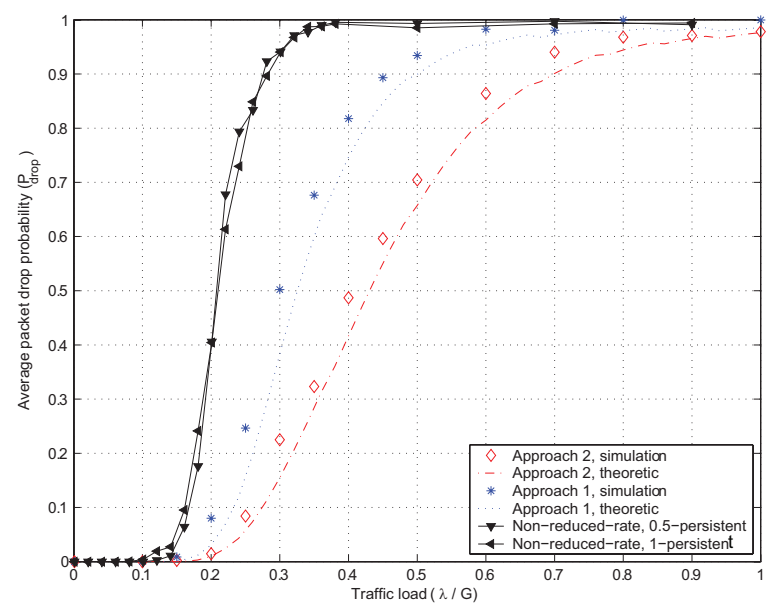

Fig. 7. Comparison of the reduced-rate retransmission and non-reduced-rate retransmission: Packet drop rate.

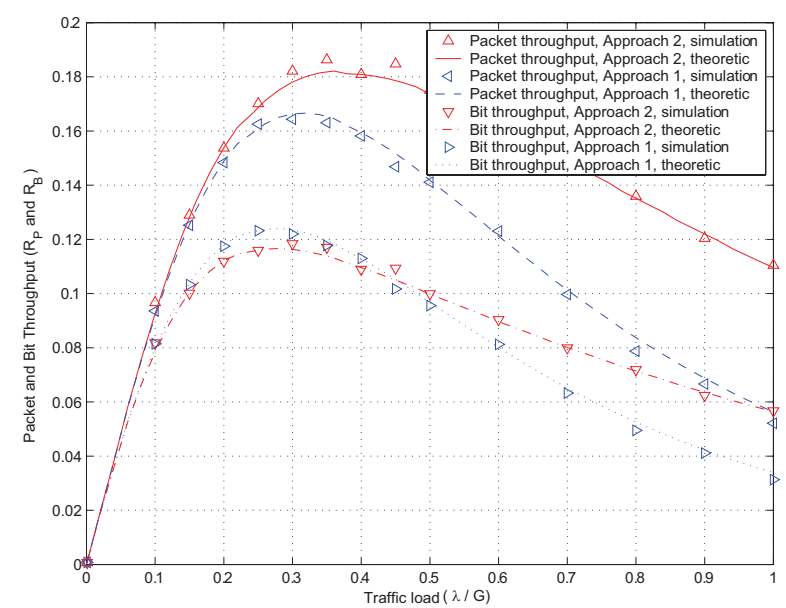

Fig. 8. Comparison of the reduced-rate retransmission and non-reduced-rate retransmission in Rayleigh fading channels: Throughput.

tolerance up to $d=3$ bit errors per packet. When fading channels are involved, the fading coefficients are drawn from a complex white Gaussian processe independently for each packet.

\section{A. One Retransmission and Non-Fading}

We first present the results of the RRR protocol under non-fading conditions in Fig. 5 for the normalized packet throughput $R_{P} / G$ and bit throughput $R_{B} / G$, in Fig. 6 for the average packet delay $D$ and in Fig. 7 for the packet drop rate $P_{\text {drop }}$, all versus the normalized traffic rate $\lambda / G$. The results are for the one-retransmission case.

For comparison, we also provide the simulation results of non-reduced-rate (with conventional random backoff) retransmission schemes with different persistent probabilities under the same system parameters. Note that for non-reduced-rate retransmission schemes, packet throughput and bit throughput are the same. It should be pointed out that persistent probability 1 corresponds to immediate retransmissions and the persistent probability 0.5 binary backoff. We can see that the two cases (persistent probability 1 and 0.5 ) perform very 


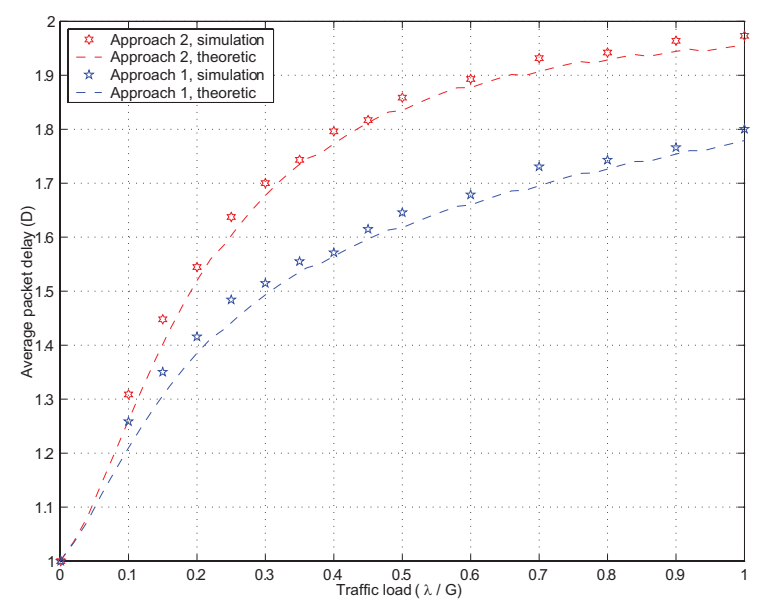

Fig. 9. Comparison of the reduced-rate retransmission and non-reduced-rate retransmission Rayleigh fading channels: Average delay.

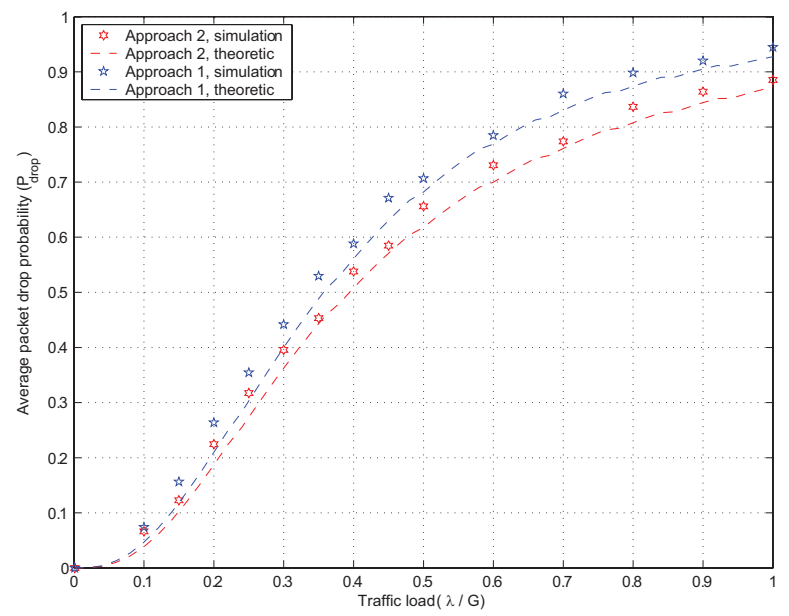

Fig. 10. Comparison of the reduced-rate retransmission and non-reduced-rate retransmission Rayleigh fading channels: Packet drop rate.

similar in terms of throughput and packet drop rate but the former one offers a lower delay than the latter. The "no retransmission" scheme is a special case of backoff when the persistent probability is 0 . It is interesting to view it as the (spreaded) slotted Aloha if we re-interpret the traffic load on the $\mathrm{x}$-axis as the aggregated load including both new arriving and retransmitted packets. This can be used as a benchmark for throughput comparison though sometime the comparison may be unfair due to the different definitions of the traffic load.

From Fig. 5, we observe that RRR Approach 2 offers the highest maximum packet throughput. Its bit throughput is slightly lower than Approach 1 and slotted Aloha with no retransmission. However, it has a higher and slower decaying tail of the (packet and bit) throughput curves in high traffic load regime, which may means a larger stable region and robustness to burstiness of traffic load. RRR Approach 1 offers an improved maximum packet throughput over the slotted Aloha scheme with no retransmissions, but its asymptotic throughput behavior as the traffic load becomes high is sim-

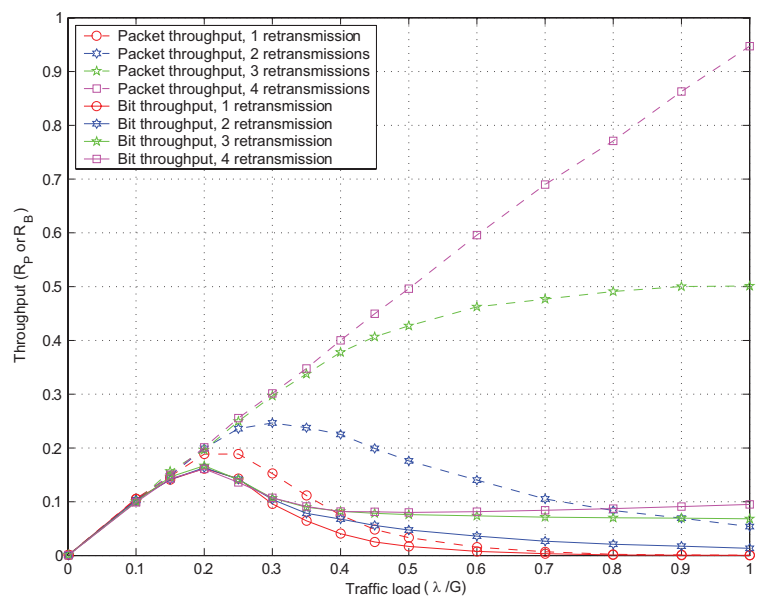

Fig. 11. Comparison of the reduced-rate retransmission scheme with different retransmission cycles: Throughput

ilar to the latter. The random backoff schemes have similar throughput performance for different persistent values. They suffer low throughput, and instability because the collapse of the throughput when traffic load becomes moderately high. It deserves notice that, compared to non-reduced-rate retransmission schemes with non-zero persistent probability, the reducedrate retransmission scheme even offers higher bit throughput, despite that part of the information bits are discarded in the rate reduction. The better throughput performance of the RRR approaches also translate to lower packet drop rate as shown in Fig. 7 from which we see that RRR Approach 2 has the lowest packet drop rate is Approach 1 is the next. As for the average packet delay, the RRR approaches outperforms. The delay is always bounded by two because maximum two transmissions are allowed. The excess delay of RRR approaches as compared to the persistent-1 backoff scheme is not as a drawback as it appears. It is due to the higher throughput of the RRR schemes so that more packets, especially the retransmitted ones are successfully transmitted. While, in the persistent-1 backoff scheme, most retransmitted ones are lost. This argument also applies to the fact that RRR Approach 2 has a longer delay that Approach 1.

Between the two RRR approaches, Approach 2 outperforms Approach 1 in terms of throughput and packet drop rate. This may be explained as follows. Comparing (3) and (4), we see that Approach 2 favors half-size packets in terms of SIRs. Its throughput gain is from reducing the interference of the full-size packets to the half-size packets by a factor of two. Approach 1, on the other hand, reduces the interference caused by half-size packets. Though the interference reduction in Approach 1 is for packets of all sizes while in Approach 2 only for half-size packets, the fact that the interference by the fullsize packets is more significant under heavy traffic conditions makes the reduction of that portion of interference more beneficial. Therefore, Approach 2 shows better performance than Approach 1 under heavy traffic conditions.

A final remark is on the consistency of the theoretical and simulation results. 


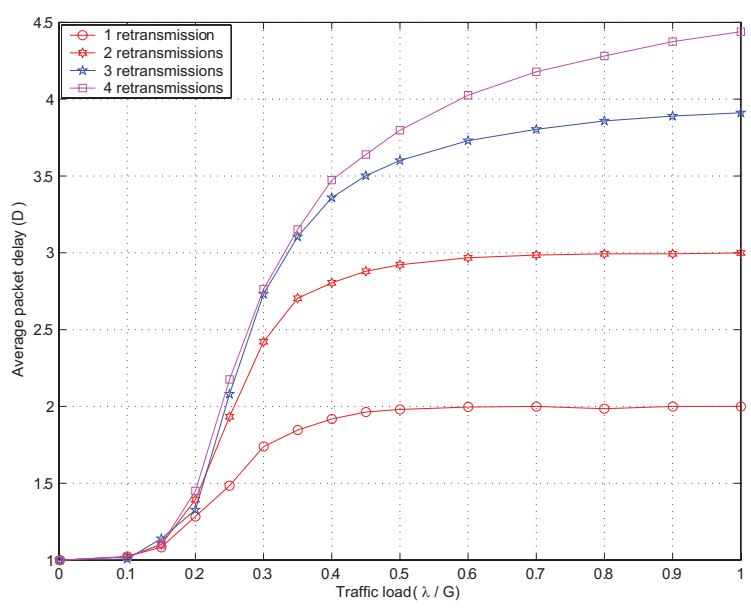

Fig. 12. Comparison of the reduced-rate retransmission scheme with different retransmission cycles: Average delay

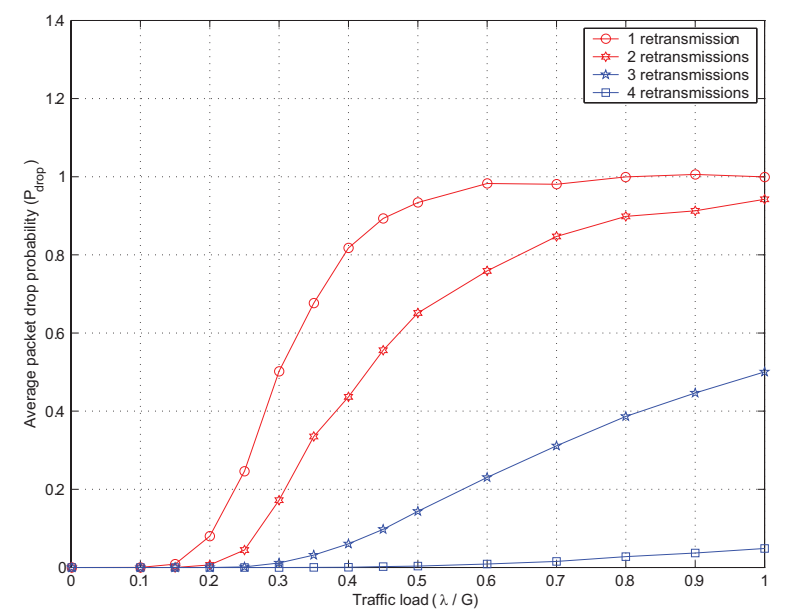

Fig. 13. Comparison of the reduced-rate retransmission scheme with different retransmission cycles: Packet drop rate

\section{B. One Retransmission and Rayleigh Fading}

Fig. 8, 9 and 10 are similar to Fig. 5, 6 and 7 except that Rayleigh fading channel is present in the analyses and simulations. Comparing the two groups of results, we see that fading-incurred capture has a negative effect on the system throughput in light traffic region but a positive effect in heavy traffic region. This observation is the consistent to that obtained in [9].

\section{Multiple Retransmissions}

The third group of results compare the performance of the RRR schemes with multiple retransmissions. Fig. 11, 12 and 13 show the throughput, delay and packet drop rate performance of RRR Approach 1 with one, two, three and four retransmissions, respectively. The following observations is obtained. Though allowing more (than one) retransmissions significantly improves the packet throughput and lowers the packet drop rate, it does not change the bit throughput very much. Indeed, in the traffic load region of practical interest, one retransmission scheme is able to offer almost the same bit throughput as those with more retransmissions. In addition, the one retransmissions scheme is superior in terms of average packet delay. Similar behavior of RRR Approach 2 can be observed.

\section{CONCLUSIONS}

In this paper, we have proposed and studied a rate adaptation scheme for spread-spectrum packet radio networks. The rate adaptation is in the form of packet-size reduction. By advantage of the widely available multi-rate source coding techniques, the source data are encoded using different code rate into packets of different sizes. The full-size packets are used for initial transmissions and smaller-size packets for retransmissions after the failure of the initial transmissions. We have proposed two approaches for the signaling of packets of reduced size: using the same processing gain while transmitting in a minislot or increasing the processing gain and transmitting over a full slot. For the first approach, since the colliding packets will be distributed into a number of minislot when retransmitted, the average interference among them due to concurrent transmissions and to other full-size packets is reduced. Therefore, a higher throughput is obtained. For the second approach, the smaller-size packets have higher processing gains, so they have a better chance to get through in collisions with full-size packets. As a result, the throughput is also increased. Our theoretical analysis and simulations have confirmed that the proposed RRR scheme offers not only a higher packet throughput but also a higher bit throughput in most traffic regime. That means that means that the information loss due to the packet-size reduction is offset by the improvement of the packet success probability due to the SIR increase. RRR Approach 2 has shown even exceptional performance superiority.

The rational of the proposed RRR technique lies in the fundamental relationship of the channel condition and information rate. In a broader context, rate control is an effective approach to provide quality of service in interference channels. Though it is usually done at higher (e.g., application, transport) layers, the RRR scheme is a solution at the medium access control layer. Since the medium access control layer is closer to the transmission medium, a quick adaptation response to the channel environment can be expected.

\section{REFERENCES}

[1] M. B. Pursley, "Throughput of frequency-hopped spread-spectrum communications for packet radio neworks," in Proc. Conf. Inform. Sci. Sys., pp. 550-556, Mar. 1983.

[2] S. L. Su and V. O. K. Li, "Performance analysis of a slotted code division multiple access (CDMA) network," in Proc. IEEE MIILCOM, pp. 23.3.1-23.3.5, Oct. 1984.

[3] M. B. Pursley, "Frequency-hop transmission for satellite packet switching and terrestrial packet radio neworks," IEEE Trans. Inform. Theory, vol. 32, pp. 652-667, Sept. 1986.

[4] D. Raychaudhuri, "Performance analysis of random access packet switched code division multiple access system," IEEE Trans. Commun., vol. 29, pp. 895-901, June 1981.

[5] R. K. Morrow, Jr., and J. S. Lehnert, "Bit-to-bit dependence in slotted DS/SSMA packet systems with random signature sequences," IEEE Trans. Commun., vol. 37, pp. 1052-1061, Oct. 1989.

[6] E. S. Sousa, J. Silvester, and T. D. Papavassiliou, "Computer-aided modeling of spread specturm packet radio networks," IEEE J. Select. Areas Commun., vol. 9, pp. 48-58, Jan. 1991. 
[7] K. Parsa, S. S. Ghassemzadeh, and S. Kazeminejad, "Systems engineering of data services in UMTS W-CDMA systems," in Proc. of ICC, vol. 5, pp. 1373-1380, June 2001.

[8] L. G. Roberts, "ALOHA packet system with and without slots and capture," Comput. Commun. Rev., pp. 28-42, Apr. 1975.

[9] A. Sheikh, Y.-D. Yao, and S. Cheng, "Throughput enhancement of direct-sequence spread-specturm packet radio neworks by adaptive power control," IEEE Trans. Commun., vol. 42, pp. 884-890, Feb. 1994.

[10] J. B. Kim and M. L. Honig, "Resource allocation for multiple classes of DS-CDMA traffic," IEEE Trans. Veh. Technol., vol. 49, pp. 506-519, Mar. 2000.

[11] R. Zhang and M. K. Tsatsanis, "A decentralised rate adaptation scheme for spread spectrum packet radio networks," in Proc. Conf. Info. Sci. Sys., pp. WA3-7 - WA3-10, Mar. 2000.

[12] K. Choi, S. K. Shin, and K. Cheun, "Adaptive processing gain CDMA networks over poisson traffic channel," IEEE Commun. Lett., vol. 6, No. 7, July 2002.

[13] V. V. Phan and S. G. Glisic, "MAC layer packet-length adaptive CLSP/DS-CDMA radio networks: Performance in flat Rayleigh fading channel," in Proc. Int. Sym. Compu. Commun., pp. 161-166, July 2002.

[14] L. Yang and L. Hanzo, "Improving the throughput of DS-CDMA systems using adaptive rate transmissions based on variable spreading factors," in Proc. IEEE VTC, Vancouver, vol. 3, pp. 1816-1820, Sep. 2002.

[15] L. Xu, X. Shen, and J. W. Mark, "Performance analysis of rate adaptation scheme for data traffic in DS-CDMA systems," in Proc. ICC, vol. 5, pp. 3372-3376, Apr. 2002.

[16] T.-H. Wu and E. Geraniotis, "CDMA with multiple chip rates for multimedia communications," in Proc. Conf. Info. Sci. Sys., pp. 992-997, Mar. 1994.

[17] D. H. Davis and S. A. Gronemeyer, "Performance of slotted ALOHA random access with delay capture and randomized time of arrival," IEEE Trans. Commun., vol. 28, pp. 703-710, May 1980

[18] K. Cheun, "Optimal arrival-time distribution for delay capture in spreadspectrum packet radio networks," in Proc. IEEE VTC, vol.2, pp. 12031207, June 1994.

[19] A. Uvilden, S. Bruhn, and R. Hagen, "Adaptive multi-rate: A speech service adapted to cellular radio network quality," in Proc. Asilomar Conf. Sig., Sys., Comput., pp. 343-347, Oct. 1998.

[20] J. Shapiro, "Embedded image coding using zerotrees of wavelet coefficients," IEEE Trans. Signal Processing, vol. 41, pp. 3445-3462, Dec. 1993.

[21] H.-J. Lee, T. Chiang, and Y.-Q. Zhang, "Scalable rate control for MPEG4 video," IEEE Trans. Circuits Syst. Video Technol., vol. 10, no. 6, pp. 878-894, Sep. 2000.

[22] M. B. Pursley, "Performance evaluation for phase-coded spreadspectrum multiple-access communication-part I: System analysis," IEEE Trans. Commun., vol. COM-25, pp. 795-799, Aug. 1977.

[23] K. Yao, "Error probability of asynchronous spread spectrum multiple access communication systems," IEEE Trans. Commun., vol. COM-25, pp. 803-809, Aug. 1977.

[24] J. Cheng and N. C. Beaulieu, "Accurate DS-CDMA bit-error probability calculation in Rayleigh fading," IEEE Trans. Wireless Commun., vol. 1, pp. 3-15, Jan. 2002.

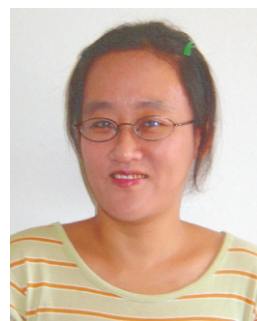

Lei Zhou (S'99) received the B.S. degree in mechanical-electronic engineering and M.E. degree in electrical engineering from Beijing Institute of Technology, Beijing China, in 1993 and 1997, respectively, and M.S. degree in computer science from Stevens Institute of Technology, Hoboken, New Jersey, in 2000. She is currently a Ph.D. candidate at the Electrical and Computer Engineering Department of Stevens Institute of Technology. She has been a graduate assistant of the Computer Science and Electrical and Computer Engineering Departments at Stevens Institute of Technology since 1998.

Her research area is wireless networking with focus on packet radio, medium access and CDMA.

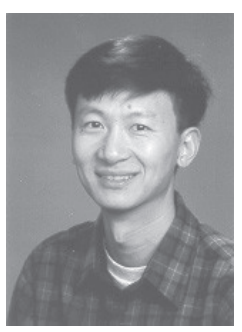

Yu-Dong Yao (S'88-M'88-SM'94) received the B.Eng. and M.Eng. degrees from Nanjing University of Posts and Telecommunications, Nanjing, China, in 1982 and 1985, respectively, and the Ph.D. degree from Southeast University, Nanjing, in 1988, all in electrical engineering. Between 1989 and 1990, he was at Carleton University, Ottawa, as a research associate working on mobile radio communications. He was with Spar Aerospace Ltd., Montreal, between 1990 and 1994, where he was involved in research on satellite communications. He was with Qualcomm Inc., San Diego, from 1994 to 2000, where he participated in research and development in wireless CDMA systems. Dr. Yao joined Stevens Institute of Technology, Hoboken, New Jersey, in 2000. He is an associate professor in the Department of Electrical and Computer Engineering and a director of Wireless Information Systems Engineering Laboratory (WISELAB). Dr. Yao holds one Chinese patent and nine US patents. He is an associate editor of IEEE Communications Letters and IEEE Transactions on Vehicular Technology, and an editor of IEEE Transactions on Wireless Communications. He is a guest editor for a special issue on wireless networks for International Journal of Communication Systems. His research interests include wireless communications and networks, spread spectrum and CDMA, and DSP for wireless systems.

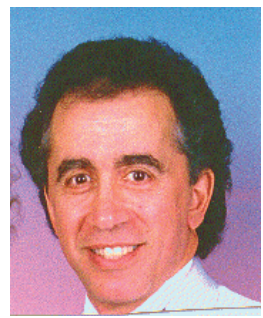

Harry Heffes (M'66-SM'82-F'90) received his B.E.E. degree from the City College of New York, New York, N.Y., in 1962, and the M.E.E.degree and the Ph.D. degree in Electrical Engineering from New York University, Bronx, N.Y., in 1964 and 1968 respectively. He joined the Department of Electrical Engineering and Computer Science in 1990. He assumed the faculty position of Professor of Electrical Engineering and Computer Science after a twenty eight year career at AT\&T Bell Laboratories. In his early years at Bell Labs he worked on the Apollo Lunar Landing Program, where he applied modern control and estimation theory to problems relating to guidance, navigation, tracking and trajectory optimization. More recently his primary concern has been with the modelling, analysis, and overload control of telecommunication systems and services. He is the author of over twenty five papers in a broad range of areas including voice and data communication networks, overload control for distributed switching systems, queuing and teletraffic theory and applications, as well as computer performance modelling and analysis.

Dr. Heffes received the Bell Labs Distinguished Technical Staff Award in 1983 and, for his work on modelling packetized voice traffic, he was awarded the IEEE Communication Society's S.O. Rice Prize in 1986. He is a fellow the IEEE for his contributions to teletraffic theory and applications. He has served as a United States Delegate to the International Teletraffic Congress and as an Associate Editor for NETWORKS: An International Journal. He is a Member of Eta Kappa Nu, Tau Beta Pi, American Men and Women of Science and is listed in Who's Who in America.

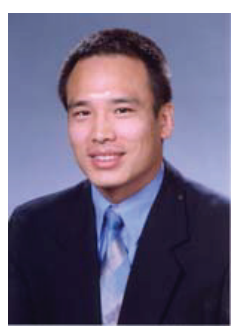

Ruifeng Zhang (S'97-M'00) received his B.S. degree from Huazhong University of Science and Technology, Wuhan, China, in 1993, M.E. degree from Beijing Institute of Technology, Beijing, China, in 1996, and Ph.D. degree from Stevens Institute of Technology, Hoboken, New Jersey, in 2000, all in electrical engineering.

$\mathrm{He}$ is an assistant professor of the Electrical and Computer Engineering Department at Drexel University, Philadelphia, Pennsylvania, which he joined in 2000. He was a research assistant and postdoctoral associate and at the Electrical and Computer Engineering Department at Stevens Institute of Technology during 1997 to 2000.

His general research interest lie in the areas of statistical signal processing and communications. Current focus of his work is on signal processing methods for wireless networks.

He received the 2002 IEEE Signal Processing Society Best Paper Award for his paper entitled "Network Assisted Diversity Multiple Access for Wireless Data Networks" (IEEE Tran. Signal Processing, 2000). He is Chair of joint SP/BT/CE Chapter, IEEE Philadelphia Section. He served in the organizing committee of ICASSP'05, the technical committee of Globecomm'04 and ICC'05. He received Peskin Award from Stevens Institute of Technology in 2000 and AT\&T and ACM Student Research Award in 1999. 